\title{
Dose-Response Effects of Bepridil in Patients With Persistent Atrial Fibrillation Monitored With Transtelephonic Electrocardiograms A Multicenter, Randomized, Placebo-Controlled, Double-Blind Study (J-BAF Study)
}

\author{
Takeshi Yamashita, MD¹; Satoshi Ogawa, MD²; Toshiaki Sato, $\mathrm{MD}^{2}$; Yoshifusa Aizawa, $\mathrm{MD}^{3}$; \\ Hirotsugu Atarashi, MD"; Akira Fujiki, MD5; Hiroshi Inoue, MD5; Makoto Ito, MD; \\ Takao Katoh, $\mathrm{MD}^{7}$; Youichi Kobayashi, MD ${ }^{8}$; Yukihiro Koretsune, $\mathrm{MD}^{9}$; Koichiro Kumagai, $\mathrm{MD}^{10}$; \\ Shinichi Niwano, MD ${ }^{11}$; Osamu Okazaki, MD ${ }^{12}$; Ken Okumura, MD ${ }^{13}$; Keijiro Saku, MD ${ }^{14}$; \\ Teruhisa Tanabe, $\mathrm{MD}^{15}$; Hideki Origasa, $\mathrm{PhD}^{16}$ for the J-BAF Investigators
}

\begin{abstract}
Background: A multicenter, randomized, placebo-controlled, double-blind trial was conducted with patients with persistent atrial fibrillation (AF) to determine the dose-response effects and safety of bepridil, using everyday transtelephonic monitorings.

Methods and Results: A total of 90 patients were randomized to receive placebo, $100 \mathrm{mg} /$ day and $200 \mathrm{mg} / \mathrm{day}$ of bepridil treatment for 12 weeks. After the treatment, those patients who converted to sinus rhythm was $3.4 \%$ in placebo, $37.5 \%$ in those who received $100 \mathrm{mg} /$ day and $69.0 \%$ in those who received $200 \mathrm{mg} /$ day, thus demonstrating a linear dose-response relationship for $\mathrm{AF}$ conversion. The conversion rate gradually reached a maximal value at $\sim 6$ weeks after initiation of bepridil. However, the $\mathrm{AF}$ recurrence rate was high $(91.7 \%$ in those receiving $100 \mathrm{mg} / \mathrm{day}$ and $75.0 \%$ in those receiving $200 \mathrm{mg} /$ day). Adverse events, presumably related to the drug, were also frequent: ventricular tachycardia in 2, QT prolongation in 4 and sinus bradycardia in 2 patients. In those patients treated with $200 \mathrm{mg} /$ day group, 1 patient died suddenly because of ventricular tachycardia.

Conclusions: This study demonstrated the dose response-relationships of bepridil for AF conversion to sinus rhythm. However, the high rate of AF recurrence and substantial drug-related adverse effects, including sudden death, raised caution about using bepridil to treat persistent AF. The balance between benefits and risks of the drug should be individualized. (Circ J 2009; 73: 1020-1027)
\end{abstract}

Key Words: Atrial fibrillation; Bepridil; Clinical trial

(Received November 9, 2008; revised manuscript received January 4, 2009; accepted January 6, 2009; released online April 9, 2009)

${ }^{1}$ The Cardiovascular Institute, ${ }^{2}$ Cardio-pulmonary Division, Department of Medicine, Keio University School of Medicine, Tokyo, ${ }^{3}$ First Department of Internal Medicine, Niigata University Graduate School of Medical and Dental Sciences, Niigata, ${ }^{4}$ Department of Internal Medicine, Nippon Medical School, Tama-Nagayama Hospital, Tama, ${ }^{5}$ Second Department of Internal Medicine, University of Toyama, Toyama, ${ }^{6}$ Department of Cardiovascular and Respiratory Medicine, Shiga University of Medical Science, Otsu, ${ }^{7}$ The First Department of Internal Medicine, Nippon Medical School, ${ }^{8}$ Third Department of Internal Medicine, Showa University School of Medicine, Tokyo, ${ }^{9}$ Cardiovascular Division, Osaka National Hospital, Osaka, ${ }^{10}$ International University of Health and Welfare, Fukuoka, "IDepartment of CardioAngiology, Kitasato University School of Medicine, Sagamihara, ${ }^{12}$ Division of Cardiology, International Medical Center of Japan, Tokyo, ${ }^{13}$ Division of Cardiology, Hirosaki University School of Medicine, Hirosaki, ${ }^{14}$ Department of Cardiology, Fukuoka University School of Medicine, Fukuoka, ${ }^{15}$ Division of Cardiology, Tokai University School of Medicine, Isehara and ${ }^{16}$ Department of Biostatistics, University of Toyama, Toyama, Japan

Mailing address: Takeshi Yamashita, MD, The Cardiovascular Insitute, 7-3-10 Roppongi, Minato-ku, Tokyo 106-0032, Japan. E-mail: yamt-tky@umin.ac.jp

All rights are reserved to the Japanese Circulation Society. For permissions, please e-mail: cj@j-circ.or.jp
$A^{\text {trial }}$ trial fibrillation (AF), which is associated with increased mortality and morbidity ${ }^{1-3}$ is a growing public health problem that has reached epidemic proportions. 4 In Japan, the population of patients with persistent/permanent $\mathrm{AF}$ is estimated to be 0.73 million people in 2005 and is likely to increase to up to 1 million people in 20106,7 For the management of these increasing AF patients, many randomized clinical studies including the AFFIRM and J-RHYTHM studies have been performed, but failed to demonstrate the superiority of the pharmacological rhythm control to rate control strategy with respect to mortality and cardiovascular events in AF patients?-10 One of the reasons for the result could be derived from inadequate antiarrhythmic effects and adverse effects of the drugs available at present. Actually, in the AFFIRM study, most patients assigned to the rhythm control strategy have been reported not to maintain sinus rhythm irrespective of aggressive treatment with amiodarone!1

\section{Editorial p 1008}

Bepridil was first developed as an anti-anginal drug, but afterwards, it has been reported to exert many electrophysi- 
ologic effects on the heart by many experimental studies ${ }^{12-18}$ In foreign countries, the antiarrhythmic effects of the drug were first examined based upon the experimental results in $1986 .{ }^{9}$ Although bepridil at a high dose of $600 \mathrm{mg} /$ day was effective for converting AF to sinus rhythm, it frequently caused remarkable QT prolongation and life-threatening arrhythmias including torsades de pointes. ${ }^{19}$ The results estimated increased risks compared with the benefits, and therefore, the drug could not be used for AF treatment in the USA and Europe. In the 2000's, several Japanese clinical studies reported that a low-dose bepridil (at 100-200 mg/day) was effective for converting persistent AF to sinus rhythm without causing any arrhythmogenic effects ${ }^{20-24}$ Since then, some Japanese specialists in cardiac electrophysiology gradually began carefully using a low-dose bepridil for persistent AF. However, those previous studies were performed in selected patients in selected institutions, and also in an open-label fashion. Therefore, it remains unknown how the results of low-dose bepridil can be applied to persistent AF in our clinical situations. Moreover, the results only analysed with periodical 12-lead electrocardiograms (ECG) could not identify the recurrence rate of AF after conversion to sinus rhythm. To validate the use of low-dose bepridil for persistent AF, it would be mandatory to solve these problems. The present study (J-BAF study) was planned as a physician-oriented clinical study to determine the dose-response effects of bepridil for conversion of persistent AF with every-day transtelephonic ECG monitoring in a multicenter, randomized, placebo-controlled, and double-blind method.

\section{Methods}

\section{Study Patients}

This clinical trial (J-BAF study) was a multicenter, randomized, placebo-controlled, parallel group study using bepridil in patients with persistent AF. In this study, persistent $\mathrm{AF}$ was defined as AF persisting for 7 days or longer ascertained on an every-day transtelephonic ECG. The following patients were excluded: (1) patients under 20 years of age; (2) patients with AF having been persisting for 1 year or longer; (3) patients within 1 month after cardiac surgery or acute myocardial infarction; (4) patients with AF presumably attributable to the following underlying disorders: sick sinus syndrome, giant left atrium (left atrial diameter $\geq 50 \mathrm{~mm}$ ), severe conduction system disturbances, hyperthyroidism, or mitral stenosis; (5) patients with a left ventricular ejection fraction of $<40 \%$ or with a Class III or IV heart failure; (6) patients with bradycardia ( $<50$ beats $/ \mathrm{min})$; (7) patients with QT interval prolongation (QTc $\geq 460 \mathrm{~ms}$ ); (8) patients with a history of syncope due to polymorphic ventricular tachycardia or antiarrhythmic drugs; (9) patients with severe hepatic or renal dysfunction; and (10) patients who were pregnancy or were lactating, and women of child-bearing potential.

\section{Study Design}

The study protocol was approved by the Institutional Review Boards at each center, and all patients gave written informed consent. During the 2 week observation period under placebo treatment, patients transmitted their ECG recordings for $30 \mathrm{~s}$ every day (at a particular time in each patient) and whenever symptoms where present using a transtelephonic monitor with a memory device (Cardiophone, Nihon-Koden, Tokyo, Japan) to the ECG center at Keio University Hospital. During this period, standard 12- lead ECG, chest X-rays, echocardiography, and blood/urine testing were also performed. After verifying that AF persisted for $\geq 7$ days and patients were eligible for the enrollment, patients were randomized to any of the following three groups: (1) placebo; (2) $100 \mathrm{mg}$; or (3) $200 \mathrm{mg} /$ day bepridil treatment. The test drugs and matching placebo, which were indistinguishable in size, weight, color and taste, were provided by Schering-Plough K.K. (Osaka, Japan). The treatment period lasted for 12 weeks, and, during this period, transtelephonic ECG monitorings were also performed every day and whenever symptoms were present to ensure precise ECG diagnosis. All ECGs were transmitted to the ECG center and also to each study center by facsimile to allow an assessment of safety for continuing the study. Twelve-lead ECG was also recorded on the first day and at $2,4,8,12$ weeks during the treatment period.

\section{Primary and Secondary End-Points}

The primary end-point of the present study was the conversion rate from persistent AF to sinus rhythm. The secondary end-points included AF recurrence after conversion to sinus rhythm, quality-of-life (QOL) improvement, and adverse events by bepridil treatment. Sinus conversion and AF recurrence were evaluated with all ECGs recorded in each patient, including transtelephonic ECGs. For QOL assessment, 2 sets of questionnaires were used: the conventional SF-36 and the AFQLQ25-27 SF-36 (the Japanese version 2) 28 consists of 8 subscales: (1) physical functioning; (2) role function-physical; (3) role function emotional; (4) bodily pain; (5) general health perceptions; (6) vitality; (7) social functioning (SF); and (8) mental health. The AFQLQ was invented by the Japanese Society of Electrocardiology as a QOL questionnaire specific for $\mathrm{AF}^{25} \mathrm{It}$ consists of 3 subscales: (1) the variety and frequency of symptoms (0-24 points); (2) the severity of symptoms (018 points); and (3) the limitations of daily and special activities and mental anxiety related to AF (0-56 points), where higher scores for each subscale indicate health status, as with SF-3625

\section{Statistical Analysis}

Data were presented as mean \pm SD. Analysis of the efficacy end-point data was performed for the full analysis set (FAS) and per protocol set (PPS), and the analysis for the FAS was taken as a primary analysis. The statistical tests were 2-tailed with a significance level of 0.05 for the comparison of groups, and a significance level of 0.2 for the homogeneity in demographic data. Demographics and baseline characteristics of each group were summarized using descriptive statistics. Continuous variables were analyzed using 1-way analysis of variance and classification variables were analyzed using the Fisher's exact test. For the number of patients, an intergroup comparison was made between the placebo group and the $200 \mathrm{mg} /$ day group, between the placebo group and the $100 \mathrm{mg} /$ day group, and between the $100 \mathrm{mg} /$ day group and the $200 \mathrm{mg} /$ day group, using the chi-squared test. With the time point at which conversion to sinus rhythm was first noted on an ECG being taken as onset of the event, an intergroup comparison was made using the log-rank test. The frequency and percentage of patients with recurrence were calculated for each treatment group, and the intergroup comparison was made using the chi-squared test. As for QOL scores, an intergroup comparison was made using the t-test between the placebo group and the $100 \mathrm{mg} /$ day group, between the placebo group and the 


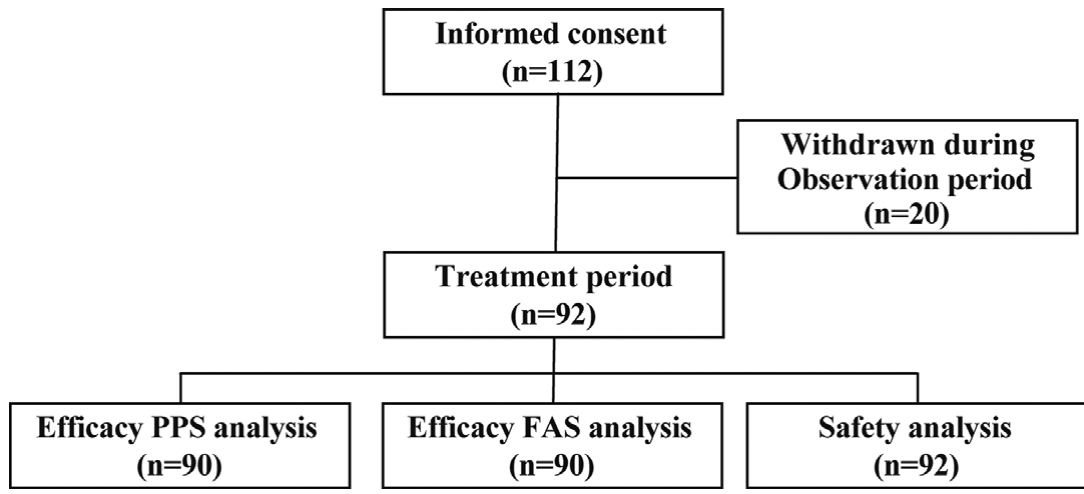

Figure 1. Study flow of PPS (per-protocol set primary efficacy analysis) and FAS (full-analysis set).

Table 1. Baseline Clinical Characteristics of the Study Patients

\begin{tabular}{lcccc}
\hline & Placebo $(\mathrm{n}=29)$ & $100 \mathrm{mg} /$ day $(\mathrm{n}=32)$ & $200 \mathrm{mg} /$ day $(\mathrm{n}=29)$ & $\mathrm{P}$ value \\
\hline Men & $25(86.2)$ & $25(78.1)$ & $24(82.8)$ & $0.739^{*}$ \\
Age (years) & $62.7 \pm 9.1$ & $63.5 \pm 13.0$ & $64.6 \pm 8.5$ & $0.802^{* *}$ \\
Weight (kg) & $68.1 \pm 13.7$ & $66.8 \pm 15.5$ & $67.8 \pm 14.4$ & $0.928^{* *}$ \\
Duration of AF (days) & $85.8 \pm 65.0$ & $108.5 \pm 92.1$ & $92.6 \pm 73.1$ & $0.507^{* *}$ \\
Underlying disease & & & & \\
$\quad$ Total & $22(75.9)$ & $21(65.6)$ & $18(62.1)$ & $0.520^{*}$ \\
$\quad$ Hypertension & $14(48.2)$ & $20(62.6)$ & $16(55.2)$ & \\
$\quad$ Valvular disease & $12(41.3)$ & $1(3.1)$ & $6(20.7)$ & \\
Ischemic heart disease & $1(3.4)$ & $5(15.7)$ & $2(6.9)$ & \\
Others & $1(3.4)$ & $3(9.4)$ & $2(6.9)$ & \\
Echocardiographic parameters & $42.6 \pm 6.6$ & $43.9 \pm 4.5$ & $43.4 \pm 5.0$ & $0.642^{* *}$ \\
$\quad$ LAD (mm) & $61.0 \pm 6.4$ & $63.5 \pm 7.3$ & $61.4 \pm 12.0$ & $0.498^{* *}$ \\
LVEF (\%) &
\end{tabular}

$\mathrm{AF}$, atrial fibrillation; $\mathrm{LAD}$, left atrial dimension; $\mathrm{LVEF}$, left ventricular ejection fraction. Data are mean \pm SD or number $(\%)$ of patients.

*Fisher's exact test, **one-way analysis of variance.

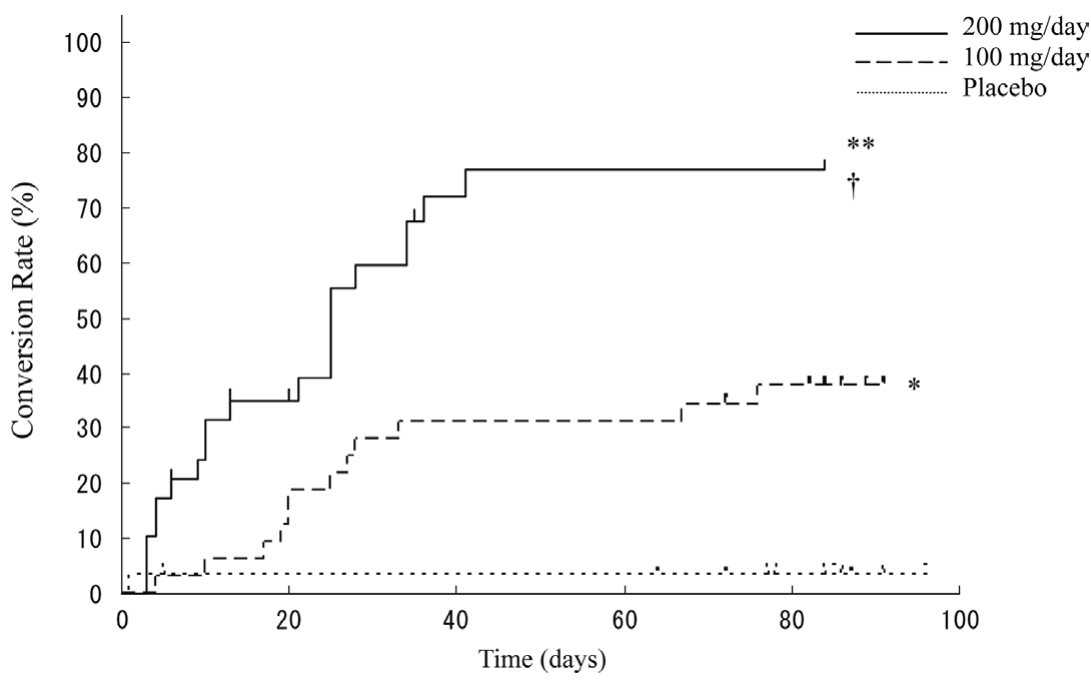

Figure 2. Conversion of persistent Atrial fibrillation (AF) to sinus rhythm. A clear doseresponse relationship of bepridil effects on $\mathrm{AF}$ conversion is observed. The conversion rate gradually increased after initiation of bepridil treatment and then reached a plateau at approximately 6 weeks after treatment.

$200 \mathrm{mg} /$ day group, and between the $100 \mathrm{mg} /$ day group and the $200 \mathrm{mg} /$ day group.

\section{Results}

\section{Patients Characteristics}

A total of 112 patients were recruited at 14 centers and 92 of them were randomly assigned to receive bepridil or placebo: 33 patients to receive $100 \mathrm{mg}$ /day of bepridil, 29 patients to receive $200 \mathrm{mg} /$ day, and 30 patients to placebo.
The remaining 20 patients were withdrawn from the study during the observation period for the following reasons (Figure 1): deviation from inclusion criteria $(n=11)$, withdrawal of informed consent $(n=4)$, deviation from exclusion criteria $(n=3)$, and other reasons $(n=1)$. Of the 92 patients, 90 were eligible for the PPS and the FAS, and their baseline clinical characteristics are presented in Table 1 . The mean age was $\sim 63$ years old, and male patients accounted for $\sim 80 \%$ of the patients. Hypertension was present in approximately half of the patients. These characteristics resembled 
those in the J-RHYTHM study recently performed in Japan. ${ }^{10}$ Although the distribution of valvular disease and ischemic heart disease was somewhat inhomogeneous among the groups, the total prevalence of underlying diseases was not significantly different. Left ventricular systolic function was well preserved and the mean left atrial dimension was $\sim 43 \mathrm{~mm}$ in each group. Twelve patients had a history of electrical cardioversion, which was reported to have failed only in 1 patient in the placebo group.

\section{Primary End-Point}

The number of patients in whom conversion to normal sinus rhythm was noted on ECG during the treatment period was 20 out of 29 patients $(69.0 \%)$ for the $200 \mathrm{mg}$ /day group, 12 out of 32 patients $(37.5 \%)$ for the $100 \mathrm{mg}$ /day group, and 1 out of 29 patients (3.4\%) for the placebo group. Analysis for superiority of $200 \mathrm{mg} /$ day and $100 \mathrm{mg}$ /day groups verified a significant effect of bepridil in converting AF to sinus rhythm, compared with the placebo (chi-square test: $\mathrm{P}<0.001$ and $\mathrm{P}=0.001$, respectively). There were also significant differences between the $200 \mathrm{mg}$ /day and $100 \mathrm{mg} /$ day groups (chi-square test: $\mathrm{P}=0.014$ ), thus demonstrating a clear dose-response relationship. An intergroup comparison with time-to-event analysis (Figure 2) also demonstrated statistically significant differences between the placebo and the $100 \mathrm{mg} /$ day groups, between the placebo and the $200 \mathrm{mg} /$ day groups, and between the $100 \mathrm{mg} / \mathrm{day}$ and the $200 \mathrm{mg} /$ day groups (log-rank test: $\mathrm{P}=0.002, \mathrm{P}<0.001$ and $\mathrm{P}=0.002$, respectively). In both the $100 \mathrm{mg} /$ day and $200 \mathrm{mg}$ /day groups, the conversion rate reached to a maximal value at approximately 6 weeks after initiation of bepridil treatment.

\section{AF Recurrence After Conversion}

Although the conversion to sinus rhythm was frequently observed by bepridil treatment, the AF recurrence rate after conversion was high, irrespective of the continued treatment. AF did not recur during the 12-week treatment period in only 5 out of 20 patients $(25.0 \%)$ that experienced sinus conversion in the $200 \mathrm{mg}$ /day group and in 1 out of 12 patients $(8.3 \%)$ in the $100 \mathrm{mg} /$ day group. Conversely, 3 patients $(15 \%)$ in the $200 \mathrm{mg} /$ day group and $5(41.7 \%)$ in the $100 \mathrm{mg} /$ day group developed persistent AF lasting $\geq 7$ days after transient conversion to sinus rhythm. In the remaining patients (12 out of 20 patients $(60 \%)$ in the $200 \mathrm{mg} /$ day group and 6 out of 12 patients $(50 \%))$, $\mathrm{AF}$ recurred with varying durations $<7$ days. In the placebo group, 1 responder experienced recurrence at 2 days after conversion to sinus rhythm. There were no significant differences in AF recurrence rate between the $100 \mathrm{mg} /$ day and the $200 \mathrm{mg} /$ day groups.

\section{QOL}

At the end of the treatment period, the $200 \mathrm{mg}$ /day group showed statistically significant improvement compared with the placebo group in 2 subscales of AFQLQ (Figure 3, $\mathrm{P}=0.001$ in the variety and frequency of symptoms and $\mathrm{P}=$ 0.002 in the severity of symptoms). In the other subscale of the limitations of daily and special activities and mental anxiety, the $200 \mathrm{mg}$ /day group showed a tendency towards improvement $(\mathrm{P}=0.052)$. Also, a statistically significant difference was observed between the $200 \mathrm{mg}$ /day and the $100 \mathrm{mg} /$ day groups with respect to the severity of symptoms $(\mathrm{P}=0.049)$. In contrast, there were no significant differences in all of the 8 subscales of the SF- $36 \mathrm{v} 2^{\mathrm{TM}}$ between the groups.
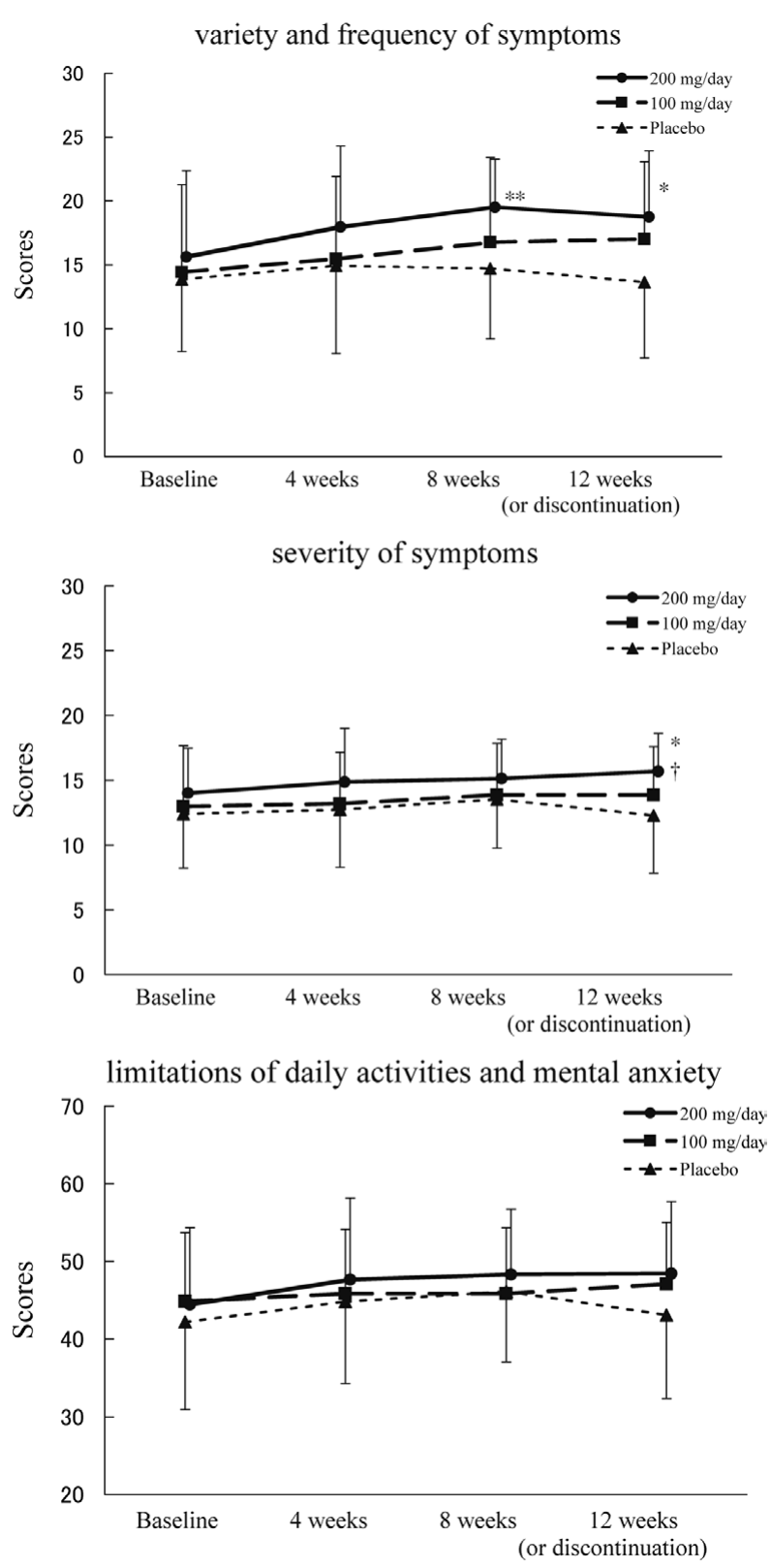

Figure 3. Clinical course of the quality-of-life questionnaire, which was specific for atrial fibrillation scores for each subscale over a 12week treatment period for patients. ${ }^{*} \mathrm{P}<0.01, * * \mathrm{P}<0.001$ vs placebo, $\dagger \mathrm{P}<0.05$ vs $100 \mathrm{mg} /$ day.

\section{Adverse Events by Bepridil (Table 2)}

The number of patients with adverse events during the study was 11 events in 7 of 29 patients $(24.1 \%)$ from the $200 \mathrm{mg} /$ day group, 8 events in 3 of 33 patients (9.1\%) from the $100 \mathrm{mg} /$ day group, and in 11 events in 6 of 30 patients $(20.0 \%)$ from the placebo group. However, the most serious case with sudden cardiac death due to ventricular tachycardia was observed in 1 patient in the $200 \mathrm{mg}$ /day group. The 59-year-old male patient with hypertension, diabetes mellitus and a history of percutaneous coronary intervention for angina developed syncope in the daytime on the $35^{\text {th }}$ day after treatment, when the automatic external defibrillator recorded ventricular tachycardia; unfortunately he could not be rescued. When he was transferred to hospital, an ECG 
Table 2. Adverse Events

\begin{tabular}{lccc}
\hline & Placebo $(\mathrm{n}=30)(\%)$ & $100 \mathrm{mg} /$ day $(\mathrm{n}=33)(\%)$ & $200 \mathrm{mg} /$ day $(\mathrm{n}=29)(\%)$ \\
\hline Ventricular tachycardia & 0 & $1(3.0)$ & $1^{*}(3.4)$ \\
QT (QTc) prolongation & 0 & 0 & $4(13.8)$ \\
Bradycardia & 0 & $1(3.0)$ & $1(3.4)$ \\
Others & $6(20.0)$ & $2(6.1)$ & $3(10.3)$ \\
\hline
\end{tabular}

*Death.

Table 3. Changes in Electrocardiographic Variables

\begin{tabular}{|c|c|c|c|c|}
\hline & Baseline & 4 weeks & 8 weeks & 12 weeks or discontinuation \\
\hline \multicolumn{5}{|c|}{ RR interval (ms) } \\
\hline Placebo & $828.5 \pm 161.0$ & $810.1 \pm 177.8$ & $810.8 \pm 185.4$ & $775.9 \pm 168.2$ \\
\hline $100 \mathrm{mg} /$ day & $805.8 \pm 146.7$ & $898.8 \pm 190.1$ & $928.1 \pm 232.6^{*}$ & $924.7 \pm 258.5^{* *}$ \\
\hline $200 \mathrm{mg} /$ day & $791.4 \pm 157.4$ & $939.0 \pm 178.7 * *$ & $942.8 \pm 153.5^{* *}$ & $963.6 \pm 174.2^{* *}$ \\
\hline \multicolumn{5}{|c|}{ QT interval (ms) } \\
\hline Placebo & $363.2 \pm 25.9$ & $361.6 \pm 26.5$ & $368.7 \pm 37.4$ & $360.3 \pm 33.0$ \\
\hline $100 \mathrm{mg} /$ day & $368.2 \pm 33.2$ & $388.5 \pm 40.6^{* *}$ & $397.2 \pm 41.7 * *$ & $394.7 \pm 49.3 * *$ \\
\hline 200 mg/day & $372.1 \pm 34.1$ & $420.7 \pm 56.7 * *, \dagger$ & $427.1 \pm 48.1 * *, \dagger$ & $442.1 \pm 64.6^{* *,+\dagger}$ \\
\hline \multicolumn{5}{|c|}{ QTc (ms) (Bazett) } \\
\hline Placebo & $403.4 \pm 36.6$ & $407.5 \pm 38.2$ & $414.8 \pm 39.7$ & $413.7 \pm 35.3$ \\
\hline $100 \mathrm{mg} /$ day & $412.7 \pm 25.6$ & $413.5 \pm 37.2$ & $417.6 \pm 39.8$ & $417.3 \pm 49.5$ \\
\hline 200 mg/day & $421.8 \pm 29.3^{*}$ & $436.0 \pm 37.6^{* *, \dagger}$ & $441.2 \pm 31.4^{*, \dagger}$ & $452.2 \pm 49.8^{* *, \dagger \dagger}$ \\
\hline \multicolumn{5}{|c|}{ QTc (ms) (Fridericia) } \\
\hline Placebo & $388.9 \pm 28.0$ & $390.7 \pm 26.2$ & $397.9 \pm 31.6$ & $394.2 \pm 27.8$ \\
\hline $100 \mathrm{mg} /$ day & $396.6 \pm 23.3$ & $404.4 \pm 32.9$ & $410.0 \pm 33.6$ & $408.7 \pm 41.9$ \\
\hline $200 \mathrm{mg} /$ day & $403.8 \pm 25.0^{*}$ & $430.3 \pm 40.7 * *, \dagger \dagger$ & $436.0 \pm 33.7 * *,+\dagger$ & $448.4 \pm 51.6 * *, \dagger \dagger$ \\
\hline
\end{tabular}

*P $<0.05,{ }^{*} \mathrm{P}<0.01$ vs Placebo, ${ }^{\dagger} \mathrm{P}<0.05,{ }^{\dagger} \mathrm{P}<0.01$ vs $100 \mathrm{mg} /$ day.

showed a sinus rhythm without QT prolongation $(\mathrm{QTc}=$ 436), and the his serum $\mathrm{K}$ and $\mathrm{Mg}$ values were 4.2 and $2 \mathrm{mmol} / \mathrm{L}$ at the event, respectively. An autopsy could not reveal any organic lesions in any organs that could explain the unexpected death, suggesting that it could have resulted from cardiac rhythm disturbances. Moreover, adverse events with presumed causal relationships to the drug were frequently observed under bepridil treatment, for example, QT (QTc) interval prolongation $>500 \mathrm{~ms}$ (4 patients in the $200 \mathrm{mg} /$ day group), ventricular tachycardia (1 patient in the $100 \mathrm{mg}$ /day group and 1 sudden death case in the $200 \mathrm{mg} /$ day group) and sinus bradycardia $<50$ beats/min ( 1 patient in both the $100 \mathrm{mg} /$ day and $200 \mathrm{mg}$ /day group). The 4 patients who showed remarkable QT prolongation after $200 \mathrm{mg}$ bepridil treatment showed significantly longer RR intervals at baseline than the other patients, although gender, age and body mass index did not differ between them. For these reasons and others, drug administration had to be discontinued in 7 patients in the $200 \mathrm{mg}$ /day group, 3 patients in the $100 \mathrm{mg} /$ day group, and 2 patients in the placebo group. As for ECG variables, changes between the baseline and the end of treatment period were dose-related in respect to RR interval, QT interval, and JT interval (Table 3, simple regression analysis: $\mathrm{P}<0.001$ in $\mathrm{RR}$ interval, $\mathrm{P}<0.001$ in $\mathrm{QT}$ interval, $\mathrm{P}=0.002$ and $\mathrm{P}<0.001$ in $\mathrm{QTc}$ (Fridericia's formula), respectively).

\section{Discussion}

\section{Major Findings}

The major findings of the present study were as follows: (1) There was a clear dose-response relationship between low-dose bepridil treatment and the conversion rate from persistent AF to sinus rhythm (38\% in the $100 \mathrm{mg} /$ day and $69 \%$ in the $200 \mathrm{mg} /$ day group). The effects were also time- dependent and almost saturated at 6 weeks after treatment; (2) The AF recurrence rate after conversion, however, was high irrespective of the continued treatment $(92 \%$ in the $100 \mathrm{mg} /$ day and $75 \%$ in the $200 \mathrm{mg}$ /day group); (3) $200 \mathrm{mg} /$ day bepridil treatment significantly relieved the variety, frequency and severity of symptoms; (4) Sudden cardiac death due to ventricular tachycardia (1 patient) and remarkable QT prolongation (4 patients) was observed in 29 patients under the 12 -week $200 \mathrm{mg}$ /day treatment.

\section{Early Studies With Bepridil}

Although bepridil was first developed as an anti-anginal drug, the drug has been shown to affect many cardiac ion channels including $\mathrm{Ca}^{2+}$ channels and also many $\mathrm{K}^{+}$channels by experimental studies!2-18 These electrophysiologic effects of bepridil led to it becoming an antiarrhythmic drug in the 1980s. The first trial with the drug for AF was reported in comparison with amiodarone ${ }^{19}$ In this study, bepridil was used at a high dose of 400-600 mg/day for persistent $\mathrm{AF}$, and the effects were compared to those produced by amiodarone, which was started at $800 \mathrm{mg} /$ day and thereafter reduced to $200 \mathrm{mg} /$ day. Nine of 14 patients were converted to sinus rhythm by the use of bepridil, while 4 of 10 were converted by amiodarone. Although the conversion rate by bepridil was remarkably high, serious adverse events were unexpectedly frequent: 2 patients developed torsades de pointes and other 4 patients experienced sustained ventricular tachycardia out of 14 patients under bepridil treatment. From the results, the authors concluded that the risks outweigh the benefits and bepridil does not offer an appreciable advantage over the established regimens or amiodarone. Actually, the FDA has not approved bepridil as an antiarrhythmic drug. 


\section{Studies With Low-Dose Bepridil}

In Japan, 15 years after the first report, clinical experiences with the drug for AF have accumulated by specialists in field of cardiac electrophysiology 20-24 They used low doses of bepridil (100-200 mg/day) for minimizing arrhythmogenic side effects in patients with persistent $\mathrm{AF}$, and found that even a low-dose bepridil was effective for the conversion of persistent AF to sinus rhythm (approximately $70 \%$ ) in selected patients without causing arrhythmogenic effects. However, at the same time, several case reports also on the side effects of using low doses of bepridil: sick sinus syndrome with torsades de pointes or interstitial pneumonitis ${ }^{29,30}$ Recently, a report with 459 AF patients has revealed that a low dose of bepridil caused adverse events in 19 patients $(4 \%)$ (QT prolongation $>0.55 \mathrm{~s}$, bradycardia, and others) including torsades de pointes in 4 patients $(0.9 \%))^{31}$ Therefore, the number of adverse effects were decreased by lowering the dose, but the fact that they occur should not be ignored.

It should be also pointed out that, in these studies with low-dose bepridil, the conversion rate and adverse events by bepridil were evaluated in an open-label fashion and in selected institutions, ${ }^{20-24}$ which could not be free from many biases. In addition, the $\mathrm{AF}$ recurrence rate after conversion to sinus rhythm is still unknown, because the evaluation was based solely upon periodical standard 12-lead ECGs. The present multicenter study was planned to overcome the limitations of the previous studies, using randomization, placebo-control, double-blindness and every-day transtelephonic ECG monitorings.

\section{Conversion and Recurrence Rate}

The present study has clearly demonstrated the doseresponse of bepridil effects on the conversion rate from persistent AF to sinus rhythm. The conversion rate by $200 \mathrm{mg} /$ day reached approximately $70 \%$, which is a high value consistent with the previous studies. In addition, the results, for the first time, clarified the treatment duration required for conversion. By using every-day transtelephonic ECG monitorings, the rate of sinus conversion gradually increased and reached a plateau at $\sim 6$ weeks after initiation of bepridil at either $100 \mathrm{mg} / \mathrm{day}$ or $200 \mathrm{mg} /$ day. Therefore, the present study not only supported the results of the previous studies but also presented the appropriate time for assessing the drug efficacy. If an inefficiency was obtained within a 6-week treatment period, it would suggest that there would be little possibility of conversion if a longer administration was used.

Although the conversion rate by the drug was high, the recurrence rate after conversion was also high irrespective of the continued treatment. Only 5 patients out of 29 patients and 1 patient out of 32 patients experienced sinus conversion without $\mathrm{AF}$ recurrence in the $200 \mathrm{mg}$ /day and $100 \mathrm{mg} /$ day treated groups, respectively. Therefore, the present study demonstrated the effectiveness for AF conversion but could not demonstrate similar effects on $\mathrm{AF}$ prevention by using bepridil. The difference between the present study results and previous studies in respect to $\mathrm{AF}$ recurrence could be the result of the methods used for detecting AF recurrence. Only a standard 12-lead ECG is well known to miss the episodes of asymptomatic AF, and thus underestimate the rate of AF recurrence.

\section{QOL}

The QOL of AF patients is important for constructing a treatment strategy for AF. In the present study, in patients with persistent AF, QOL scores by AFQLQ were relatively high at baseline, as compared with a recent study involving $\mathrm{AF}$ patients undergoing catheter ablation. Nevertheless, $200 \mathrm{mg} /$ day of bepridil significantly improved the 2 subscales of AFQLQ: the frequency and the variety, and severity of symptoms. These effects could be derived from sinus conversion by bepridil and also the heart rate control by the drug, which is known to have $\mathrm{Ca}^{2+}$ channel-blocking effects! ${ }^{2,13}$ In contrast, it should be remembered that the drug could not relieve the limitations of daily and special activities and mental anxiety related to AF, and did not improve SF-36 QOL scores. These results might be attributable to a high AF recurrence rate even under a continued treatment.

\section{Adverse Events}

As previous studies have demonstrated, arrhythmogenic effects by bepridil have been a great concern with its induced QT prolongation. Actually, in the present study, the drug administration had to be discontinued due to QT prolongation in 4 out of 29 patients (14\%) undergoing a $200 \mathrm{mg} /$ day treatment. This discontinuation rate seems somewhat higher than that in previous studies ${ }^{20-24}$ and the difference might be attributed to the randomization and double-blindness of the present study.

The most important fact was that sudden cardiac death due to ventricular tachycardia was observed in 1 patient (3.4\%, confidence interval $0.6-19.5 \%$ ) in the $200 \mathrm{mg} /$ day group. The history of ischemic heart disease of this patient might be related to this unexpected event. However, it should be noted that the lowest value of the confidence interval of the event rate almost corresponded with the rate of polymorphic ventricular tachycardia $(0.9 \%)$ caused by low-dose bepridil, which was reported recently 29 Although polymorphic ventricular tachycardia is well known to be one of the bepridil-induced adverse events, a previous study ${ }^{19}$ has also reported that monomorphic ventricular tachycardia could also be caused by bepridil administration, which was almost identical to our case. A cohort study with Japanese AF patients has demonstrated that the cardiovascular mortality of AF patients is estimated to be approximately $1.3 \%$ / year ${ }^{32}$ and the mortality rate in the J-RHYTHM study was also low (0.6\%/year) $!^{10}$ Based on these low mortalities of Japanese AF patients, the sudden death observed in the present study should be emphasized and in turn should also limit the use of this low-dose bepridil treatment. At the same time, tolerability for continuing the drug would be another concern, because 7 (24\%) of patients under the $200 \mathrm{mg} /$ day treatment group could not continue the study for various reasons including QT prolongation.

Primarily, many recent large clinical trials have demonstrated that there were no significant differences in the prognosis of patients with persistent AF between rhythm control and rate control strategies ${ }^{8,9}$ The present study results with bepridil used in a rhythm control strategy should be interpreted in these situations, and the unignorable adverse events caused by the drug also supported the recent clinical evidence. Therefore, the present study does not promote the use of low-dose bepridil as treatment for persistent AF and rather demonstrates the requirement for even more caution, especially when the drug usage is necessary in some selected patients. 


\section{Study Limitations}

The present study has several limitations. First, it used a relatively small number of the patients. However, we believe that the small number of patients did not mask the adverse effects by bepridil in the present study. Second is that the results were obtained only in patients with persistent $\mathrm{AF}$ and from a relatively short follow-up period. Therefore, the results should not be extrapolated to paroxysmal AF or to long-term bepridil treatment, because the study could not demonstrate the effectiveness and safety for $\mathrm{AF}$ prevention. Thirdly, it should be pointed out that bepridil dosages require careful reconsideration if it is to be used for AF treatment. Lastly, the present study enrolled only patients with preserved systolic function, and most of the patients were male. Therefore, the effects of bepridil in AF patients with decreased $\mathrm{EF}$, congestive heart failure or in older female patients are totally unknown, particularly in regards to the adverse events. The present study could underestimate the adverse events in real-world medical practice, and therefore should not support bepridil usage for these patients.

\section{Conclusions}

The present study has demonstrated dose-dependent effectiveness of bepridil for conversion of persistent $\mathrm{AF}$, but also identified the high rate of AF recurrence after conversion and also substantial adverse events by its arrhythmogenic effects. Although the drug might be an effective tool for AF conversion in some selected cases, the balance between benefits and risks is still arguable and, therefore, the usage should be individualized and closely monitored with caution, because the mortality of Japanese AF patients is quite low even under a standard rate control strategy.

\section{References}

1. Benjamin EJ, Wolf PA, D'Agostino RB, Silbershatz H, Kannel WB, Levy D. Impact of atrial fibrillation on the risk of death: The Framingham Heart Study. Circulation 1998; 8: 946-952.

2. Vidaillet H, Granada JF, Chyou PH, Maassen K, Ortiz M, Pulido JN, et al. A population-based study of mortality among patients with atrial fibrillation or flutter. Am J Med 2002; 113: 365-370.

3. Krahn AD, Manfreda J, Tate RB, Mathewson FA, Cuddy TE. The natural history of atrial fibrillation: Incidence, risk factors, and prognosis in the Manitoba Follow-Up Study. Am J Med 1995; 98: 476484.

4. Le Heuzey JY, Paziaud O, Piot O, Said MA, Copie X, Lavergne T, et al. Cost of care distribution in atrial fibrillation patients: The COCAF study. Am Heart J 2004; 147: 121 - 126.

5. Stewart S, Murphy NF, Walker A, McGuire A, McMurray JJ. Cost of an emerging epidemic: An economic analysis of atrial fibrillation in the UK. Heart 2004; 90: 286-292.

6. Ohsawa M, Okayama A, Sakata K, Kato K, Itai K, Onoda T, et al. Rapid increase in estimated number of persons with atrial fibrillation in Japan: An analysis from national surveys on cardiovascular diseases in 1980, 1990 and 2000. J Epidemiol 2005; 15: 194-196.

7. Inoue H, Fujiki A, Origasa H, Ogawa S, Okumura K, Kubota I, et al. Prevalence of atrial fibrillation in the general population of Japan: An analysis based on periodic health examination. Int J Cardiol 2008 (in press).

8. Wyse DG, Waldo AL, DiMarco JP, Domanski MJ, Rosenberg Y, Schron EB, et al. A comparison of rate control and rhythm control in patients with atrial fibrillation. $N$ Engl J Med 2002; 347: 18251833.

9. Van Gelder IC, Hagens VE, Bosker HA, Kingma JH, Kamp O, Kingma T, et al. A comparison of rate control and rhythm control in patients with recurrent persistent atrial fibrillation. $N$ Engl J Med 2002; 347: 1834-1840.

10. Ogawa S, Yamashita T, Yamazaki T, Aizawa Y, Atarashi H, Inoue H, et al. Optimal treatment strategy for patients with paroxysmal atrial fibrillation: J-RHYTHM Study. Circ J 2009; 73: 242-248.
11. AFFIRM First Antiarrhythmic Drug Substudy Investigators. Maintenance of sinus rhythm in patients with atrial fibrillation: An AFFIRM substudy of the first antiarrhythmic drug. J Am Coll Cardiol 2003; 42: $20-29$.

12. Yatani A, Brown AM, Schwartz A. Bepridil block of cardiac calcium and sodium channels. J Pharmacol Exp Ther 1986; 237: 9-17.

13. Cohen CJ, Spires S, Van Skiver D. Block of T-type Ca channels in guinea pig atrial cells by antiarrhythmic agents and $\mathrm{Ca}$ channel antagonists. J Gen Physiol 1992; 100: 703-728.

14. Kamiya K, Kodama I, Lu Z. Effects of Bepridil on Ikr and Iks of rabbit ventricular myocytes. Environment Med 2002; 46: 95-97.

15. Kobayashi S, Reien Y, Ogura T, Saito T, Masuda Y, Nakaya H. Inhibitory effect of Bepridil on hKv1.5 channel current: Comparison with Amiodarone and E-4031. Eur J Pharmacol 2001; 430: 149157.

16. Berger F, Borchard U, Hafner D. Effects of the calcium entry blocker Bepridil on repolarizing and pacemaker currents in sheep cardiac Purkinje fibres. Naunyn Schmiedebergs Arch Pharmacol 1989; 339: $638-646$.

17. Hara Y, Nakaya H. SD-3212, a new class I and IV antiarrhythmic drug: A potent inhibitor of the muscarinic acetylcholine-receptoroperated potassium current in guinea-pig atrial cells. Br J Pharmacol 1995; 116: $2750-2756$

18. Sato T, Costa AD, Saito T, Ogura T, Ishida H, Garlid KD, et al. Bepridil, an antiarrhythmic drug, opens mitochondrial KATP channels, blocks sarcolemmal KATP channels, and confers cardioprotection. J Pharmacol Exp Ther 2006; 316: 182-188.

19. Perelman MS, McKenna WJ, Rowland E, Krikler DM. A comparison of Bepridil with Amiodarone in the treatment of established atrial fibrillation. Br Heart J 1987; 58: 339-344.

20. Fujiki A, Tsuneda T, Sugao M, Mizumaki K, Inoue H. Usefulness and safety of Bepridil in converting persistent atrial fibrillation to sinus rhythm. Am J Cardiol 2003; 92: 472-475.

21. Miyaji K, Tada H, Fukushima Kusano K, Hashimoto T, Kaseno K, Hiramatsu S, et al. Efficacy and safety of the additional Bepridil treatment in patients with atrial fibrillation refractory to class I antiarrhythmic drugs. Circ J 2007; 71: 1250-1257.

22. Fujiki A, Tsuneda T, Sakabe M, Nakagawa K, Mizumaki K, Hirai T, et al. Maintenance of sinus rhythm and recovery of atrial mechanical function after cardioversion with Bepridil or in combination with aprindine in long-lasting persistent atrial fibrillation. Circ J 2004; 68: 834-839.

23. Nakazato Y, Yasuda M, Sasaki A, Iida Y, Kawano Y, Nakazato K, et al. Conversion and maintenance of sinus rhythm by Bepridil in patients with persistent atrial fibrillation. Circ J 2005; 69: 44-48.

24. Fujiki A, Sakamoto T, Iwamoto J, Nishida K, Nagasawa H, Mizumaki $\mathrm{K}$, et al. Pharmacological cardioversion of persistent atrial fibrillation with and without a history of drug-resistant paroxysmal atrial fibrillation. Circ J 2006; 70: 1138-1141.

25. Yamashita T, Kumagai K, Koretsune Y, Mitamura H, Okumura K, Ogawa S, et al. A new method for evaluating quality of life specific to patients with atrial fibrillation: Atrial fibrillation Quality of Life Questionnaire (AFQLQ). Jpn J Electrocardiol 2003; 23: 332-343 (in Japanese).

26. Yamashita T, Komatsu T, Kumagai K, Uno K, Niwano S, Fijiki A, et al. Internal consistency and reproducibility of Atrial fibrillation Quality of Life Questionnaire (AFQLQ). Jpn J Electrocardiol 2005; 25: 488494 (in Japanese).

27. Yamashita T, Ogawa S, Aizawa Y, Atarashi H, Inoue H, Ohe T, et al on behalf of the J-RHYTHM Investigators. Investigation of the optimal treatment strategy for atrial fibrillation in Japan: The JRHYTHM (Japanese Rhythm Management Trial for Atrial Fibrillation) Study design. Circ J 2003; 67: 738-741.

28. Fukuhara S, Suzukamo Y. Manual of SF-36v2 $2^{\mathrm{TM}}$ Japanese version. Tokyo: Public Health Research Foundation, 2004.

29. Ohsawa M, Tanaka F, Kunugita F, Saito H, Nakamura M, Shiroto T, et al. A case of sick sinus syndrome that developed torsades de pointes, pacing failure and sensing failure during administration of Bepridil. Jpn Heart J 2003; 44: 783-788.

30. Gaku S, Naoshi K, Teruhiko A. A case of Bepridil induced interstitial pneumonitis. Heart 2003; 89: 1415.

31. Yasuda M, Nakazato Y, Sasaki A, Kawano Y, Nakazato K, Tokano T, et al. Clinical evaluation of adverse effects during Bepridil administration for atrial fibrillation and flutter. Circ J 2006; 70: 662-666.

32. Suzuki S, Yamashita T, Ohtsuka T, Sagara K, Uejima T, Oikawa Y, et al. Prevalence and prognosis of patients with atrial fibrillation in Japan: A prospective cohort of Shinken Database 2004. Circ J 2008; 72: $914-920$. 


\section{Appendix}

J-BAF Investigators

The following centers and investigators participated in this multicenter trial.

Hirosaki University School of Medicine (Aomori): Ken Okumura, Atsushi

Iwasa, Masaomi Kimura.

Keio University School of Medicine (Tokyo): Satoshi Ogawa.

Nippon Medical School (Tokyo): Takao Katoh, Yoshinori Kobayashi, Yasushi Miyauchi, Yu-ki Iwasaki.

Nippon Medical School Tama-Nagayama Hospital (Tokyo): Hirotsugu Atarashi, Eitaro Kodani, Makoto Matsumoto.

The Cardiovascular Institute Hospital (Tokyo): Takeshi Yamashita, Koichi Sagara, Takayuki Ohtsuka.

Showa University School of Medicine (Tokyo): Youichi Kobayashi, Taku Asano, Mitsuharu Kawamura.
International Medical Center of Japan (Tokyo): Osamu Okazaki.

Tokai University School of Medicine (Kanagawa): Teruhisa Tanabe, Koichiro Yoshioka, Yoshiaki Deguchi.

Kitasato University School of Medicine (Kanagawa): Shinichi Niwano, Masaru Yuge.

Niigata University Graduate School of Medical and Dental Sciences (Niigata): Yoshifusa Aizawa, Hiroshi Furushima.

Faculty of Medicine,University of Toyama (Toyama): Akira Fujiki, Koichi Mizumaki.

Shiga University of Medical Science (Shiga): Makoto Ito, Takashi Ashihara, Hideki Itoh, Yoshihisa Sugimoto.

Osaka National Hospital (Osaka): Yukihiro Koretsune.

Fukuoka University Hospital (Fukuoka): Keijiro Saku, Matsumoto Naomichi, Tomoo Yasuda. 